ARTICLE

Received 12 Apr 2014 | Accepted 31 Jul 2014 | Published 9 Sep $2014 \quad$ DOI: 10.1038/ncomms5867

\title{
Dislocation motion and grain boundary migration in two-dimensional tungsten disulphide
}

\author{
Amin Azizi ${ }^{1,2, \star}$, Xiaolong Zou ${ }^{3, \star}$, Peter Ercius ${ }^{4}$, Zhuhua Zhang ${ }^{3}$, Ana Laura Elías ${ }^{2,5}$, Néstor Perea-López ${ }^{2,5}$, \\ Greg Stone ${ }^{1}$, Mauricio Terrones ${ }^{1,2,5,6}$, Boris I. Yakobson ${ }^{3} \&$ Nasim Alem ${ }^{1,2}$
}

Dislocations have a significant effect on mechanical, electronic, magnetic and optical properties of crystals. For a dislocation to migrate in bulk crystals, collective and simultaneous movement of several atoms is needed. In two-dimensional crystals, in contrast, dislocations occur on the surface and can exhibit unique migration dynamics. Dislocation migration has recently been studied in graphene, but no studies have been reported on dislocation dynamics for two-dimensional transition metal dichalcogenides with unique metal-ligand bonding and a three-atom thickness. This study presents dislocation motion, glide and climb, leading to grain boundary migration in a tungsten disulphide monolayer. Direct atomic-scale imaging coupled with atomistic simulations reveals a strikingly low-energy barrier for glide, leading to significant grain boundary reconstruction in tungsten disulphide. The observed dynamics are unique and different from those reported for graphene. Through strain field mapping, we also demonstrate how dislocations introduce considerable strain along the grain boundaries and at the dislocation cores.

\footnotetext{
${ }^{1}$ Department of Materials Science and Engineering, Materials Research Institute, Pennsylvania State University, University Park, Pennsylvania 16802, USA

${ }^{2}$ Center for Two Dimensional and Layered Materials, Pennsylvania State University, University Park, Pennsylvania 16802, USA. ${ }^{3}$ Department of Materials Science and Nano-Engineering, and the Richard Smalley Institute, Rice University, Houston, Texas 77005, USA. ${ }^{4}$ National Center for Electron Microscopy, Lawrence Berkeley National Laboratory, Berkeley, California 94720, USA. ${ }^{5}$ Department of Physics, Pennsylvania State University, University Park, Pennsylvania 16802, USA. ${ }^{6}$ Department of Chemistry, Pennsylvania State University, University Park, Pennsylvania 16802, USA. * These authors contributed equally to this work. Correspondence and requests for materials should be addressed to N.A. (email: nua10@psu.edu) or to B.I.Y. (email: biy@rice.edu).
} 
$\mathrm{D}$ islocations can significantly modulate the electronic properties of crystals by introducing states within their band gap ${ }^{1}$. They can act as nucleation sites for the onset of deformation, when subjected to stress ${ }^{2-4}$. When exposed to chemical functional groups, defects can also behave as highly reactive sites and efficiently trap different molecules ${ }^{5,6}$. A rich variety of dislocations have recently been observed in $2 \mathrm{D}$ crystals such as graphene, hexagonal boron nitride and transition metal dichalcogenides (TMDs) ${ }^{7-12}$. Due to the high surface to volume ratio of $2 \mathrm{D}$ crystals, dislocations can lead to unique crystal geometries by introducing strain ${ }^{9}$. Dislocation migration in threedimensional bulk crystals occurs when a large number of atoms move simultaneously along or perpendicular to its Burgers vector, leading to glide or climb, respectively. In contrast to bulk crystals, dislocation migration in $2 \mathrm{D}$ crystals can take place through the movement of few atoms near the dislocation core and the massive movement of atoms is not required. Because of the unusual geometry of $2 \mathrm{D}$ crystals, dislocation glide occurs by the movement of atoms on the surface, in which atomic diffusion typically has a lower-energy barrier when compared with the bulk. Graphene is an example of a $2 \mathrm{D}$ crystal, in which a dislocation glide can take place through Stone-Wales bond rotation ${ }^{7,9,13,14}$. Unlike graphene, $\mathrm{MX}_{2}$ TMDs consist of metal (for example, $\mathrm{M}=\mathrm{Mo}, \mathrm{W}, \mathrm{Nb}$ and so on) atoms sandwiched by chalcogen atoms (for example, $\mathrm{X}=\mathrm{S}, \mathrm{Se}, \mathrm{Te}$ ) establishing partial ionic bonds (see Supplementary Fig. 1). This elemental heterogeneity can give rise to a completely different migration behaviour as homo-nuclear bond formation is not energetically favourable.

In this study, we use aberration-corrected scanning transmission electron microscopy (STEM) imaging to probe the dislocations and their migration mechanisms leading to grain boundary migration in $\mathrm{WS}_{2}$ monolayer. We study the grain boundary structure, stability and dynamics resulting from the sample-beam interactions in chemical vapor deposition (CVD) grown monolayer $\mathrm{WS}_{2}$ (ref. 15). With a combination of experiment and theory, this investigation elucidates for the first time the dislocation-motion mechanisms in a hetero-elemental $\mathrm{WS}_{2} 2 \mathrm{D}$ crystal and the local strain field associated with its dislocations.

\section{Results}

Grain boundary structure in a monolayer $\mathrm{WS}_{2}$ and its electronic structure. Figure la,b illustrates two tilt grain boundaries (shown by the arrows) with $12^{\circ}$ and $22^{\circ}$ misorientation angles in a monolayer of $\mathrm{WS}_{2}$ exhibiting several $6 \mid 8$ edge dislocations. At the larger tilt of $22^{\circ}$, a higher dislocation density is observed along the grain boundary that leads to higher local strain. Such level of strain near the grain boundaries can lead to a slight warping of the grains out of the $\mathrm{WS}_{2}$ monolayer plane, resulting in the contrast loss of sulphur atoms in the annular dark field (ADF) STEM images as a result of local distortion (see Supplementary Figs 2 and 3). Both grain boundaries are dominantly comprised of $6 \mid 8$ edge dislocations (Fig. 1d,e), which are derived from $5 \mid 7$ defects by the insertion of a two-atom column of S between the $\mathrm{W}-\mathrm{W}$ bonds ${ }^{16}$. In particular, the $6 \mid 8$ dislocations are predicted to be more favourable under S-rich conditions when compared with $5 \mid 7$ dislocations in TMDs ${ }^{16} .6 \mid 8$ dislocations were also observed in $\mathrm{MoS}_{2}$ (ref. 17) but have not been observed in any other 2D materials. The distance between dislocation cores along the $12^{\circ}$ grain boundary is about $1 \mathrm{~nm}$ (see Supplementary Fig. 4), which is in close agreement with the simulated structure of the $9^{\circ}$ grain boundary composed of $6 \mid 8 \mathrm{~s}$
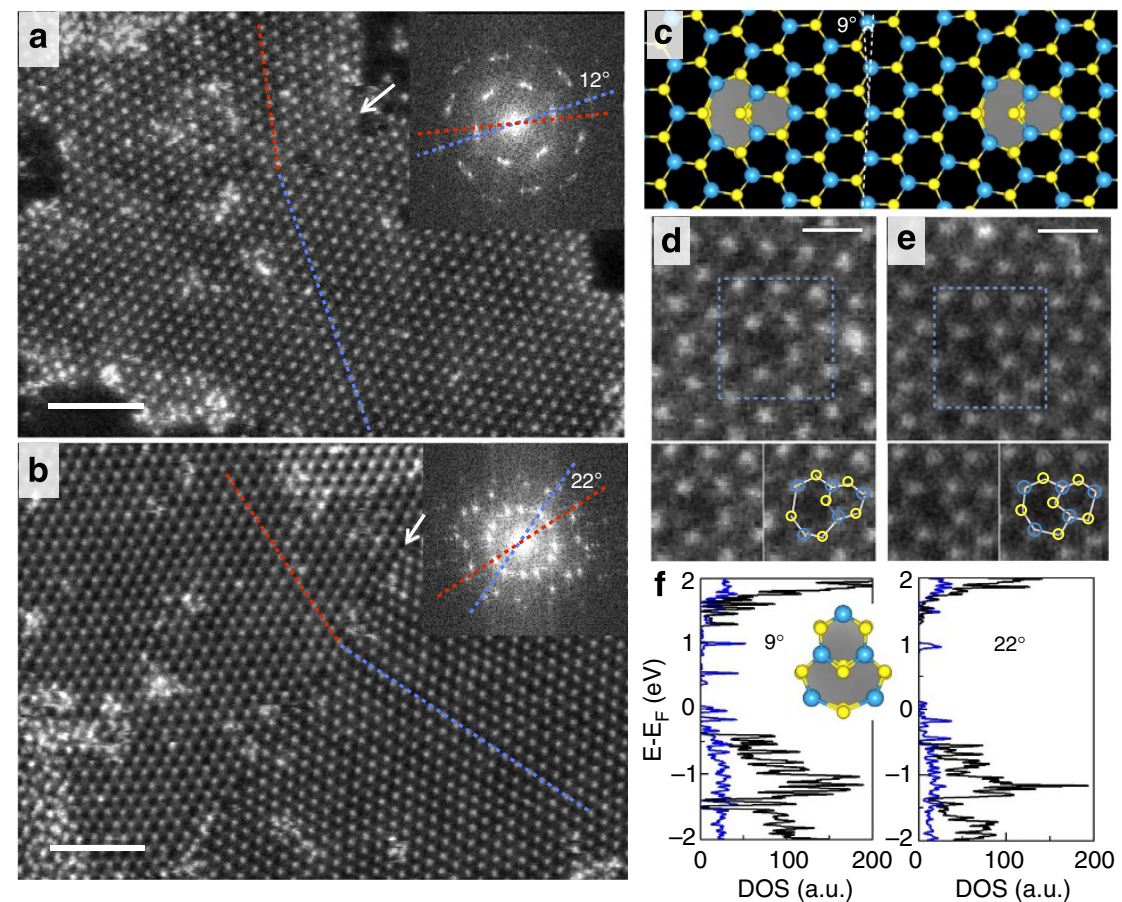

Figure 1 | Low- and high-angel grain boundary structure of the monolayer $\mathbf{W S}_{\mathbf{2}}$ with its electronic structure. (a) ADF-STEM image of a tilt grain boundary in a single-layer $\mathrm{WS}_{2}$ sheet and the corresponding FFT, with a misorientation angle of $12^{\circ}$ between two grains as marked by dashed lines. Scale bar, $2 \mathrm{~nm}$. (b) ADF-STEM image and the corresponding FFT of a grain boundary with a $22^{\circ}$ misorientation angle. Scale bar, $2 \mathrm{~nm}$. (c) Atomic structure model calculated from first principles for a $9^{\circ}$ grain boundary composed of $6 \mid 8$ dislocations. (d,e) The magnified regions of a $6 \mid 8$ dislocation core in $12^{\circ}$ and $22^{\circ}$ grain boundaries, respectively. The lower images highlight the position of $\mathrm{W}$ and $\mathrm{S}$ atoms and the $6 \mid 8$ dislocation structure. The scale bar is $5 \AA$. (f) Total electronic density of states (grey line) for the $9^{\circ}$ (left) and $22^{\circ}$ (right) grain boundaries composed of $6 \mid 8$ structures, respectively. The blue line shows the states contributed by atoms forming the dislocations. 
(Fig. 1c). Electronic structure analysis from first-principles calculations indicates that $6 \mid 8$ dislocations introduce a series of localized states within the band gap of $\mathrm{WS}_{2}$ monolayer (Fig. 1f), which may serve as sinks for charge carriers to undermine the electron transport in $\mathrm{WS}_{2}$. More importantly, the $6 \mid 8$ dislocations are nonmagnetic, in contrast to the magnetic $5 \mid 7$ dislocations in $\mathrm{TMDs}^{18}$. This indicates that the incorporation of two sulphur atoms in the $5 \mid 7$ dislocations can quench the radical electronic states of the $5 \mid 7$ dislocations and therefore electronically stabilize the $6 \mid 8$ dislocations. This distinctly differentiates TMDs from graphene $^{19-21}$ and hexagonal boron nitride ${ }^{10}$, in which $5 \mid 7$ dislocations are also observed.

Strain-field mapping of the grain boundaries in the monolayer $\mathbf{W S}_{2}$. Dislocations at grain boundaries can locally introduce considerable levels of strain that can further accommodate dislocation movements in $2 \mathrm{D}$ crystals. Here we use geometric phase analysis $^{22}$ to map the strain fields at the dislocation cores in the low- and high-angle grain boundaries. A symmetric strain matrix was used to obtain strain fields for $\varepsilon_{x x}, \varepsilon_{x y}, \varepsilon_{y y}$ and rotation $x y$ (Fig. 2). Figure $2 \mathrm{a}, \mathrm{f}$ shows superimposed images of the $12^{\circ}$ and $22^{\circ}$ grain boundaries (originally depicted in Fig. 1a,b, respectively) and their $\varepsilon_{x y}$ strain maps, indicating a considerable amount of shear strain present at the dislocation cores. Figure $2 b-$ e and g-j shows $\varepsilon_{x x}, \varepsilon_{x y}, \varepsilon_{y y}$ strain and rotation $x y$ maps for the $12^{\circ}$ and $22^{\circ}$ grain boundaries, respectively. The $12^{\circ}$ grain boundary shows shear strain $\left(\varepsilon_{x y}\right)$ in the range of $5-25 \%$ (corresponding to distances ranging from 0.50 to $0.035 \mathrm{~nm}$ from the dislocation core), whereas a considerably higher strain ranging from 5 to $58 \%$ (corresponding to distances ranging from 0.50 to $0.035 \mathrm{~nm}$ from the dislocation core) was found for the $22^{\circ}$ grain boundary. In addition, the $22^{\circ}$ grain boundary shows larger $\varepsilon_{x x}, \varepsilon_{y y}$ strain fields

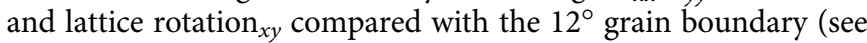
also real space strain analysis in Supplementary Fig. 5). Such high-strain fields associated with the dislocations can lead to unique migration dynamics and reconstruction mechanisms that need to be further explored.

In this report, we show a qualitatively new phenomenon on the structural dynamics of dislocations in 2D TMDs: the migration of $6 \mid 8$ dislocations in a monolayer $\mathrm{WS}_{2}$ at room temperature and low-accelerating voltage $(80 \mathrm{kV})$ for both low- and high-angle grain boundaries.

Dislocation migration in a $12^{\circ}$ grain boundary of the monolayer $\mathbf{W S}_{2}$. Supplementary Movie 1 shows a series of ADF-STEM images where dislocation migration leads to grain boundary motion in a monolayer of $\mathrm{WS}_{2}$. Selected snapshots from the observed atomic-scale dynamics can be seen in Fig. 3. The consecutive frames of this movie are $5 \mathrm{~s}$ apart, showing the dislocation migration in a low-angle grain boundary $\left(12^{\circ}\right)$. Figure $3 \mathrm{a}, \mathrm{b}$ shows glide of the marked dislocation to the bottom right, along its Burgers vector. Figure $3 \mathrm{c}, \mathrm{d}$ reveals the same dislocation several seconds later, which moves to the bottom right again, through a glide along its Burgers vector marked by an arrow in Fig. 3c. These dynamics are analysed by considering the dislocation core with respect to a stationary vacancy as a reference point in every frame (see Supplementary Fig. 6). By comparing individual frames of the movie, $S$ atoms are observed to be very dynamic and mobile around the dislocation cores. This dynamic behaviour could lead to dislocations exhibiting S-rich or -deficient conditions as well as different atomic arrangements ${ }^{23}$. In particular, S-rich/deficient structures were observed at the dislocation core, which can be achieved by either thermodynamically controlling the chemical potential ${ }^{16}$ or by knock-on displacement of atoms from the incident electron beam ${ }^{17,24,25}$. A S-rich structure with two sulphur atoms at the dislocation core can form a $6 \mid 8$ dislocation (see Fig. 3a and Supplementary Figs 7A and 8C), whereas a S-deficient structure (Fig. $3 \mathrm{c}$ and Supplementary

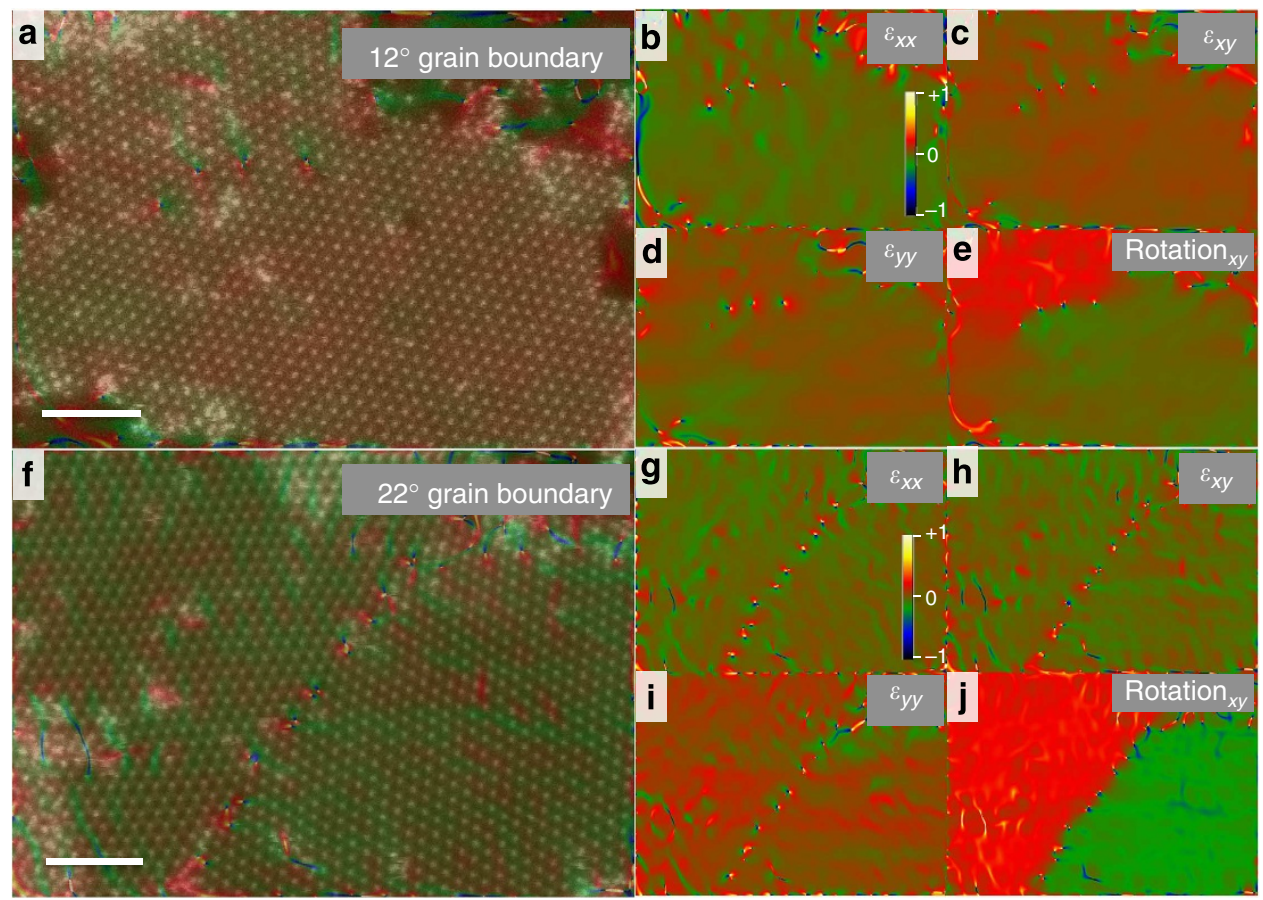

Figure 2 | Strain-field mapping of the low- and high-angle grain boundaries of the monolayer $\mathbf{W S}_{\mathbf{2}}$. (a) A superimposed image of the $12^{\circ}$ grain boundary and its $\epsilon_{x y}$ strain map. Scale bar, $2 \mathrm{~nm}$. (b) $\varepsilon_{x x}(\mathbf{c}) \varepsilon_{x y}(\mathbf{d}) \varepsilon_{y y}$ and (e) rotation ${ }_{x y}$ maps for the $12^{\circ}$ grain boundary. (f) A superimposed image of the $22^{\circ}$ grain boundary and its corresponding strain field $\varepsilon_{x y}$. Scale bar, $2 \mathrm{~nm}$. (g) $\varepsilon_{x x \prime}(\mathbf{h}) \varepsilon_{x y \prime}(\mathbf{i}) \varepsilon_{y y}$ and $(\mathbf{j})$ rotation $x y$ maps for the $22^{\circ}$ grain boundary. The strain colour scale (shown in Fig. $2 b, g$ ) corresponds to a range from -1 (black) to +1 (white), in which the boundary between red and green signifies 0 . 

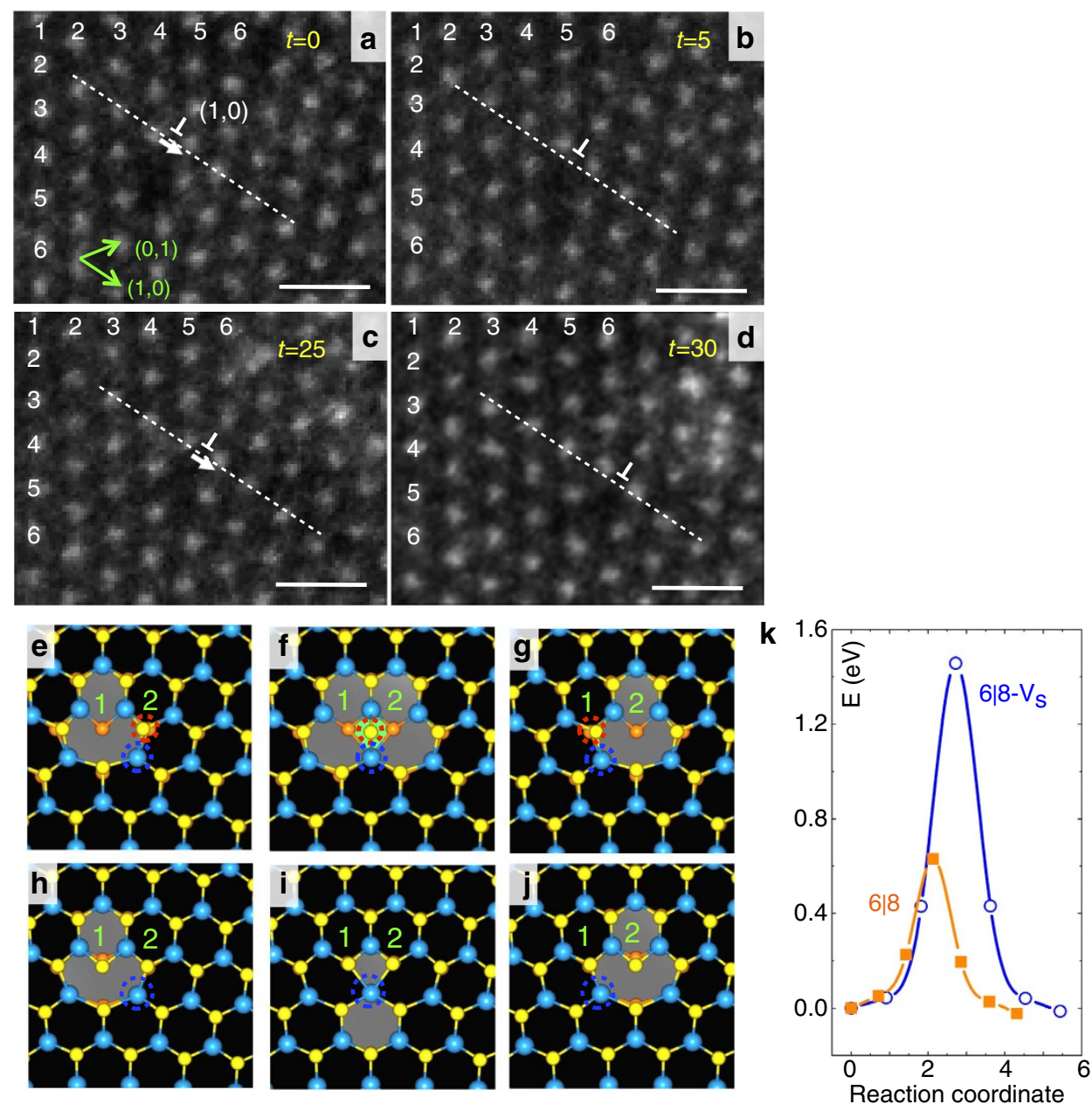

Figure 3 | Dislocation migration in a $\mathbf{1 2}^{\circ}$ grain boundary. Dislocation glide along its Burgers vector $(1,0)$ on its glide plane (shown by dashed lines) from $\mathbf{a}$ to $\mathbf{b}$. Several seconds later the same dislocation glides again along $(1,0)$ from $\mathbf{c}$ to $\mathbf{d}$. (e-g) Structural models for the migration of a $6 \mid 8$ dislocation with one $\mathrm{S}$ vacancy $\left(6 \mid 8-V_{S}\right)$. (h-j) $6 \mid 8$ with initial, saddle-point and final structures in the left, middle and right panels, respectively. (k) Minimum energy paths during migration for two dislocation cores. The scale bars are $6 \AA$.

Figs $7 \mathrm{~B}$ and $8 \mathrm{~F}$ ) with one $\mathrm{S}$ atom missing at the dislocation core can result in the formation of a $6 \mid 8-\mathrm{V}_{\mathrm{S}}$ dislocation (6|8 dislocation with one sulphur vacancy). Here the formation of $S$ vacancies in TMDs results from the electron beam-sample interactions at $80 \mathrm{kV}$ (ref. 24). When using this particular electron beam energy, we are on the edge of the knock-on threshold, where $\mathrm{W}$ atoms mainly remain in the lattice, whereas several $S$ vacancies can be created at the edges, dislocations and within the defect-free region of the monolayer. Sulphur adatoms arising from this knock-on process can display mobility on the surface of the $\mathrm{WS}_{2}$ monolayer, especially around the dislocation cores and edges. In addition, other adatoms, such as carbon, can exhibit similar mobility and interaction with the dislocations, which should be further investigated. Such dynamics are the key component for transforming the dislocation structure leading to dislocation glide and grain boundary migration ${ }^{9,26}$.

First-principles calculations were carried out in order to gain a better understanding of the dislocation migration mechanism observed in $\mathrm{WS}_{2}$ monolayer. Both dislocation structures, $6 \mid 8$ and $6 \mid 8-V_{S}$, can be formed through interaction of the $5 \mid 7$ dislocations with $S$ interstitials ${ }^{16,27}$. Figure $3 e-j$ depicts the mechanism for the migration of the $6 \mid 8-\mathrm{V}_{\mathrm{S}}$ and $6 \mid 8$ dislocations. In this figure, the orange atoms located at the dislocation core show the $\mathrm{S}$ atoms on the bottom layer, whereas the yellow atoms indicate $S$ atoms on the top layer. The migration of $6 \mid 8-\mathrm{V}_{\mathrm{S}}$ is displayed in Fig. $3 \mathrm{e}-\mathrm{g}$ with only one $S$ atom at site 1 . Our calculations suggest dislocation migration starts when one of the top $S$ atoms at site 2 (red dashed circle) moves to site 1 . This movement is accompanied by the circled $\mathrm{W}$ atom simultaneously gliding and disconnecting from the $S$ atom at site 2, thus creating a bond with the $S$ atom at site 1 . The concurrent movements of $W$ and $S$ atoms lead to a significant structural distortion in the saddlepoint structure (Fig. 3f) that results in a migration barrier of about $1.4 \mathrm{eV}$. This energy barrier is considerably lower than the diffusion energy barrier for a single $\mathrm{S}$ vacancy $\left(\mathrm{V}_{\mathrm{S}}\right)$, which is estimated to be $2.3 \mathrm{eV}$ for $\mathrm{MoS}_{2}$ of the same lattice ${ }^{28}$, highlighting the important role of the dislocations and their interaction with $S$ vacancies in mediating both the dislocation and $S$ migration in TMDs through the coupled displacement of $\mathrm{W}$ atoms. Without the $S$ vacancy in the dislocation core (Fig. $3 \mathrm{~h}-\mathrm{j}$ ), the dislocation migration barrier further drops to $0.6 \mathrm{eV}$ (Fig. 3k). Under this condition, the lattice undergoes a much smaller structural distortion, as the $\mathrm{S}$ sublattice recovers and only a single $\mathrm{W}$ atom is involved during the dislocation glide through direct bond breaking and reconnection. As shown in Fig. $3 \mathrm{~h}-\mathrm{j}$, the circled W atom breaks its bonds with $\mathrm{S}$ atoms at site 2 , and reconnects with $\mathrm{S}$ atoms at site 1 . As the glide proceeds, a W-oriented $4 \mid 6$ structure forms at the saddle point with the (circled) W-S bond 
length increased by about $20 \mathrm{pm}$ compared with those forming hexagons (Fig. 3i). This results in a considerable drop in the energy barrier for dislocation glide under S-rich conditions. Such a low-migration barrier in monolayer $\mathrm{WS}_{2}$ is already similar to bubbles diffusing in liquids, and in sharp contrast to graphene, where there is an energy barrier of $5-10 \mathrm{eV}$ for dislocation glide through bond rotation ${ }^{9,13,14}$. Other TMD systems can exhibit similar migration mechanisms to $\mathrm{WS}_{2}$ because of their similar bonding and atomic structure.

Dislocation migration in a $22^{\circ}$ grain boundary of the monolayer $\mathbf{W S}_{2}$. Supplementary Movie 2 shows dislocation migration in a high-angle $\left(22^{\circ}\right)$ grain boundary in monolayer $\mathrm{WS}_{2}$. A sequence of ADF-STEM images from the dislocation cores can be followed in Fig. 4a-d (see Supplementary Fig. 9 for Burgers circuit analysis and Supplementary Fig. 10 for the relaxed dislocation structures). The grain boundary in this area is composed of both $6 \mid 8$ dislocations with $(1,0)$ Burgers vector and $4 \mid 6$ dislocations with $(1,-1)$ (equivalent to $(0,1))^{16}$ Burgers vector (see Fig. 4e). In the high-angle grain boundaries with a higher density of dislocations, the migration of different dislocations can be coupled together, resulting in different migration paths. Figure 4a,b reveals two consecutive frames of the grain boundary $5 \mathrm{~s}$ apart. The top and bottom $6 \mid 8$ dislocations glide two and one steps along the $(1,0)$ direction, respectively. Meanwhile, the $4 \mid 6$ dislocation glides one step along the $(1,-1)$ direction and then climbs one step in the $(0,-1)$ direction. This migration results from the successive diffusion of few atoms locally near the $6 \mid 8$ and $4 \mid 6$ composite dislocations (see Supplementary Fig. 11). Here, for the first time, we show climb and glide as two representative migration paths for dislocation migration in 2D TMDs materials.

Figure $4 \mathrm{c}$ shows the same dislocation after $10 \mathrm{~s}$, in which two side-by-side $6 \mid 8$ dislocations are formed as a result of further rearrangement of atoms around the composite dislocations during the dislocation climb. The two side-by-side $6 \mid 8$ dislocations further merge through glide and form a $6|8| 8$ dislocation with $(2,0)$ Burgers vector, as shown in Fig. $4 d$. This dislocation glide is energetically favourable and leads to a significant energy release (about $-2.4 \mathrm{eV}$ ). Such a large dislocation can create a high-strain field and is considered as an ideal site for $\mathrm{S}$ interstitials or other adatoms, such as $\mathrm{C}$. The simultaneous climb and glide observed here is complex and different from the migration behaviour at low-angle grain boundaries, where dislocation migration is identified as an isolated glide process.

\section{Discussion}

Using aberration-corrected electron microscopy coupled with first principles calculations this study reveals unique and fascinating dislocation structures along the grain boundaries and their local strain fields in a monolayer of $\mathrm{WS}_{2}$. Strain analysis shows large levels of strain along the grain boundaries and locally at the dislocation cores. Through a series of ADF-STEM images, the movement of individual atoms have been successfully probed at the grain boundaries and within the dislocation cores. Owing to the unique geometry of $2 \mathrm{D}$ crystals, dislocations reside at the crystal surface and can easily interact with the surface atoms. Highly mobile $S$ atoms can interact with the dislocation cores and form different derivative dislocation structures with different migration mechanisms involving the rearrangement of the local
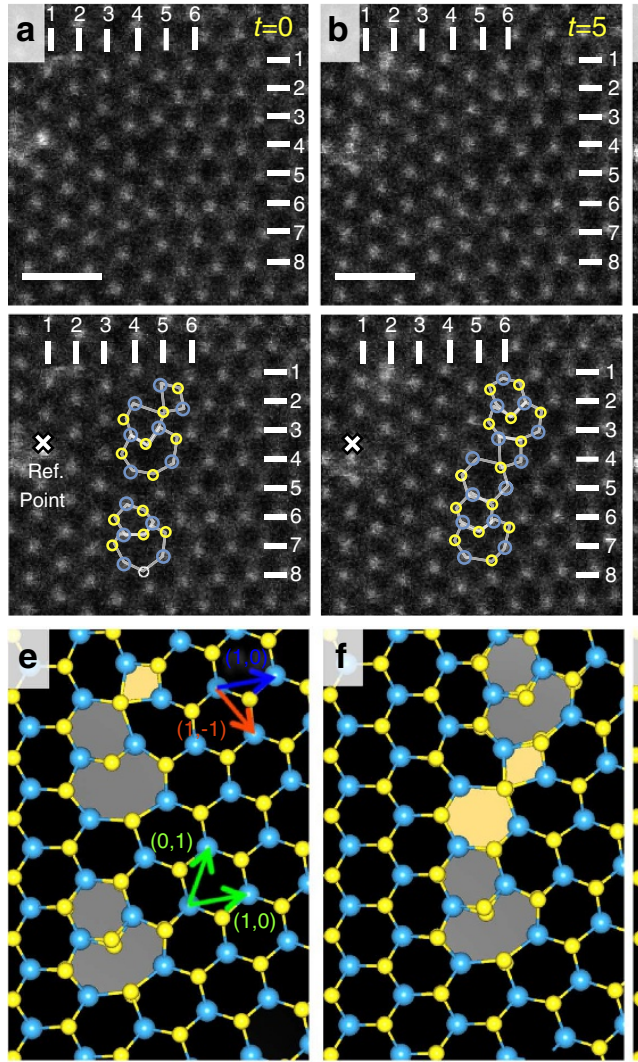
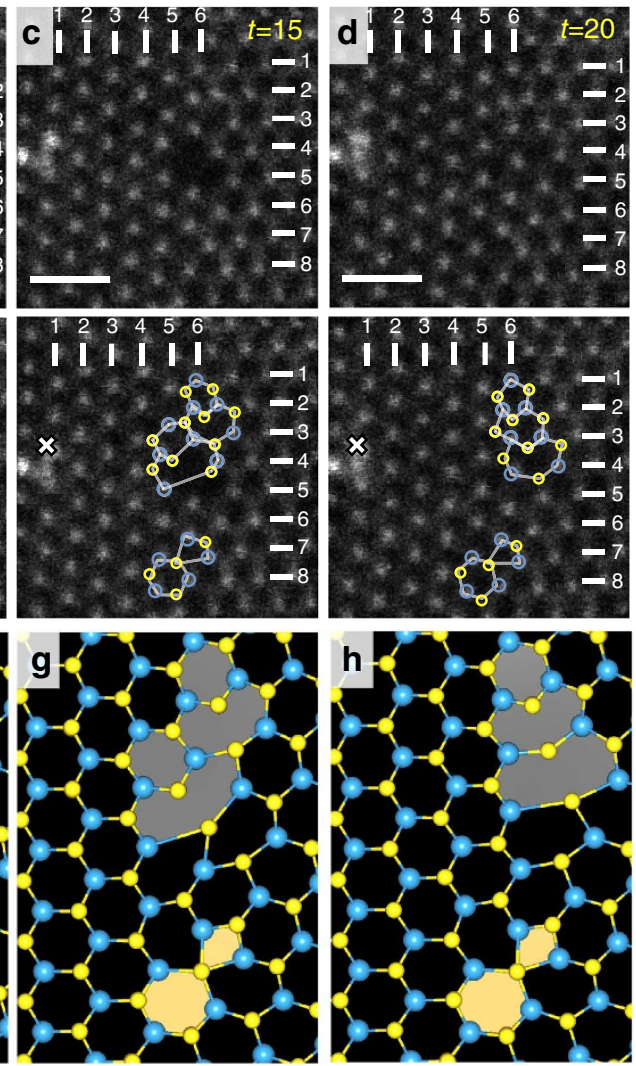

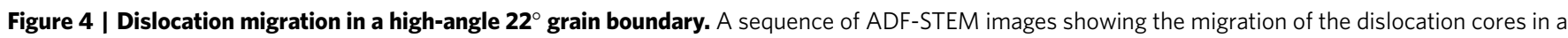

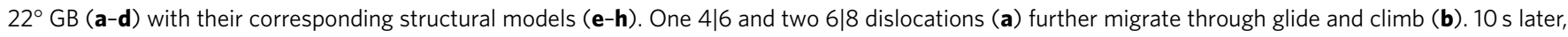
two side-by-side $6 \mid 8 \mathrm{~s}$ (c) recombine to form a $6|6| 8$ dislocation (d). The green arrows in e show the crystal vectors for the pristine lattice, whereas the blue and orange arrows indicate two Burgers vectors $(1,0)$ and $(1,-1)$ (equivalent to $(0,1)$ ) for $6 \mid 8$ and $4 \mid 6$, respectively. The scale bars are $7 \AA$. 
$\mathrm{W}$ and $\mathrm{S}$ atoms around the defect. We observe such dynamics to have a strikingly low-energy barrier leading to significant grain boundary reconstruction in TMDs, despite their complex lattice structure. Controlling defects such as grain boundaries and dislocations using electron or ion irradiation have important implications in the design of $2 \mathrm{D}$ materials with desired physicochemical properties, and this account has provided important pieces of information regarding the transformation of defects and their dynamics.

\section{Methods}

Synthesis. $\mathrm{WS}_{2}$ synthesis was carried out through a two-step process ${ }^{15}$. Si wafers with a thermally deposited $\mathrm{SiO}_{2}$ layer 285 -nm thick were thoroughly cleaned with a piranha solution at $80^{\circ} \mathrm{C}$ for at least $40 \mathrm{~min}$. After piranha treatment, the $\mathrm{Si} / \mathrm{SiO}_{2}$ wafers were loaded in the chamber of a physical vapour deposition apparatus, PVD75 (Kurt J. Lesker). A 1-nm layer of $\mathrm{WO}_{3}$ was thermally evaporated and deposited on the wafers at low pressures $\left(10^{-5}-10^{-6} \mathrm{Torr}\right)$. The $\mathrm{Si} / \mathrm{SiO}_{2} / \mathrm{WO}_{3}$ wafers were subsequently loaded into a quartz reaction tube for thermal treatment under a S environment. The pressure in the chamber was set to $35 \mathrm{mTorr}$ for $10 \mathrm{~min}$ and of ultra high purity (UHP) $\mathrm{Ar}$ (30 s.c.c.m.) was then allowed to flow into the reaction tube, reaching a pressure of $250 \mathrm{mTorr}$. The split furnace was then heated to $850^{\circ} \mathrm{C}$.

Transmission electron microscopy sample preparation. $\mathrm{The}_{\mathrm{WS}}$ films were transferred onto Quantifoil Au TEM grids by using a poly(methyl methacrylate) (PMMA)-assisted technique. The films were coated with a thin layer of poly(methyl methacrylate), which was cured at room temperature for at least $2 \mathrm{~h}$. The samples were then immersed in hydrofluoric acid for a few seconds in order to lift off the PMMA-WS ${ }_{2}$ films. The film was removed and immersed in five beakers of deionized water, subsequently. The film was then picked up by the TEM grid from the surface of the water and was dried for a few hours. The PMMA layer was dissolved in acetone and isopropanol. To remove the remaining contaminants, the grids were annealed under Ar atmosphere at $400^{\circ} \mathrm{C}$ for $4 \mathrm{~h}$. The residue of the PMMA formed a defected graphene film that served as a support during imaging of the flake.

Imaging of grain boundary structure and dynamics. TEAM I at the National Center for Electron Microscopy at Lawrence Berkeley National Laboratory was used for this study. ADF-STEM images of $\mathrm{WS}_{2}$ were recorded using $80 \mathrm{kV}, 70 \mathrm{pA}$ of beam current, $30 \mathrm{mrad}$ convergence semi-angle and an ADF semi-angle of $\sim 45 \mathrm{mrad}$. Image pixel sizes were $25 \mathrm{pm}$ and dwell time was $15 \mu$ s to provide sufficient time resolution to study grain boundary and defect migration dynamics.

Density functional theory calculations. Density functional theory (DFT) calculations have been performed with the Perdew-Burke-Ernzerhof parametrization ${ }^{29}$ of the generalized gradient approximation and projector-augmented wave potentials $^{30,31}$, using the Vienna $A b$-initio Simulation Package ${ }^{32,33}$. The vacuum layer thickness is chosen to be larger than $10 \AA$ to keep the spurious interaction negligible by adopting a supercell approach. Based on the plane-wave-based total energy minimization ${ }^{34}$, all structures are fully relaxed until the force on each atom was less than $0.01 \mathrm{eV}^{-1}$. The climbing image nudged elastic band method ${ }^{35}$ was used to calculate the activation barrier with a convergence criterion of $0.1 \mathrm{eV} \AA^{-1}$, which shows a negligible barrier difference when compared with those with a more stringent criterion of $0.05 \mathrm{eV}^{-1}$. All the structures have been constructed in nanoribbon configurations, and the strain effects have been examined (see Supplementary Figs 12 and 13). Although the tensile strain is shown to have little influence on the migration barrier (Supplementary Fig. 12), shear strain results in the distortion of $6 \mid 8$ cores, as observed in TEM images (Supplementary Fig. 13).

\section{References}

1. Batson, P. Atomic and electronic structure of a dissociated $60^{\circ}$ misfit dislocation in GexSi(1-x). Phys. Rev. Lett. 83, 4409-4412 (1999).

2. Zobelli, A. et al. Defective structure of BN nanotubes: from single vacancies to dislocation lines. Nano Lett. 6, 1955-1960 (2006).

3. Alem, N. \& Dravid, V. P. Correlative deformation mechanisms in $\mathrm{NixCo}_{1-\mathrm{x}} \mathrm{O} /$ $\mathrm{ZrO}_{2}(\mathrm{CaO})$ directionally solidified eutectic composites with a confined metallic interphase. Acta Mater. 56, 4378-4389 (2008).

4. Tang, D.-M. et al. Revealing the anomalous tensile properties of WS2 nanotubes by in situ transmission electron microscopy. Nano Lett. 13, 1034-1040 (2013).

5. Salehi-Khojin, A. et al. Polycrystalline graphene ribbons as chemiresistors. Adv. Mater. 24, 53-57 (2012)

6. Hajati, Y. et al. Improved gas sensing activity in structurally defected bilayer graphene. Nanotechnology 23, 505501 (2012).
7. Lehtinen, O., Kurasch, S., Krasheninnikov, a. V. \& Kaiser, U. Atomic scale study of the life cycle of a dislocation in graphene from birth to annihilation. Nat. Commun 4, 2098 (2013).

8. Butz, B. et al. Dislocations in bilayer graphene. Nature 505, 533-537 (2014).

9. Warner, J. H. et al. Dislocation-driven deformations in graphene. Science 337, 209-212 (2012).

10. Gibb, A. L. et al. Atomic resolution imaging of grain boundary defects in monolayer chemical vapor deposition-grown hexagonal boron nitride. J. Am. Chem. Soc. 135, 6758-6761 (2013).

11. Najmaei, S. et al. Vapour phase growth and grain boundary structure of molybdenum disulphide atomic layers. Nat. Mater. 12, 754-759 (2013).

12. Van der Zande, A. M. et al. Grains and grain boundaries in highly crystalline monolayer molybdenum disulphide. Nat. Mater. 12, 554-561 (2013).

13. Ding, F., Jiao, K., Wu, M. \& Yakobson, B. Pseudoclimb and dislocation dynamics in superplastic nanotubes. Phys. Rev. Lett. 98, 075503 (2007).

14. Dumitrica, T., Hua, M. \& Yakobson, B. I. Symmetry-, time-, and temperaturedependent strength of carbon nanotubes. Proc. Natl Acad. Sci. USA 103, 6105-6109 (2006).

15. Elías, A. L. et al. Controlled synthesis and transfer of large-area WS2 sheets: from single layer to few layers. ACS Nano 7, 5235-5242 (2013).

16. Zou, X., Liu, Y. \& Yakobson, B. I. Predicting dislocations and grain boundaries in two-dimensional metal-disulfides from the first principles. Nano Lett. 13, 253-258 (2013).

17. Zhou, W. et al. Intrinsic structural defects in monolayer molybdenum disulfide. Nano Lett. 13, 2615-2622 (2013).

18. Zhang, Z., Zou, X., Crespi, V. H. \& Yakobson, B. I. Intrinsic magnetism of grain boundaries in two-dimensional metal dichalcogenides. ACS Nano 7, 10475-10481 (2013).

19. Huang, P. Y. et al. Grains and grain boundaries in single-layer graphene atomic patchwork quilts. Nature 469, 389-392 (2011).

20. Meyer, J. C. et al. Direct imaging of lattice atoms and topological defects in graphene membranes. Nano Lett. 8, 3582-3586 (2008).

21. Kim, K. et al. Grain boundary mapping in polycrystalline graphene. ACS Nano 5, 2142-2146 (2011).

22. Hÿtch, M. J., Snoeck, E. \& Kilaas, R. Quantitative measurement of displacement and strain fields from HREM micrographs. Ultramicroscopy 74, 131-146 (1998).

23. Krasheninnikov, A. V. \& Banhart, F. Engineering of nanostructured carbon materials with electron or ion beams. Nat. Mater. 6, 723-733 (2007).

24. Komsa, H.-P. et al. Two-dimensional transition metal dichalcogenides under electron irradiation: defect production and doping. Phys. Rev. Lett. 109, 035503 (2012).

25. Zan, R. et al. Control of radiation damage in MoS2 by graphene encapsulation. ACS Nano 7, 10167-10174 (2013).

26. Girit, C. O. et al. Graphene at the edge: stability and dynamics. Science 323, 1705-1708 (2009).

27. Hirth, J. P. \& Lothe, J. Theory of Dislocations (Wiley, 1982).

28. Komsa, H.-P., Kurasch, S., Lehtinen, O., Kaiser, U. \& Krasheninnikov, A. V. From point to extended defects in two-dimensional $\mathrm{MoS}_{2}$ : Evolution of atomic structure under electron irradiation. Phys. Rev. B 88, 035301 (2013).

29. Perdew, J. P., Burke, K. \& Ernzerhof, M. Generalized gradient approximation made simple. Phys. Rev. Lett. 77, 3865-3868 (1996).

30. Blöchl, P. E. Projector augmented-wave method. Phys. Rev. B 50, 17953-17979 (1994).

31. Kresse, G. \& Joubert, D. From ultrasoft pseudopotentials to the projector augmented-wave method. Phys. Rev. B 59, 1758-1775 (1999).

32. Kresse, G. \& Furthmüller, J. Efficiency of ab-initio total energy calculations for metals and semiconductors using a plane-wave basis set. Comput. Mater. Sci. 6, 15-50 (1996).

33. Kresse, G. \& Furthmüller, J. Efficient iterative schemes for ab initio totalenergy calculations using a plane-wave basis set. Phys. Rev. B 54, 11169-11186 (1996).

34. Ihm, J., Zunger, A. \& Cohen, M. L. Momentum-space formalism for the total energy of solids. J. Phys. C Solid State Phys. 12, 4409-4422 (1979).

35. Henkelman, G., Uberuaga, B. P. \& Jónsson, H. A climbing image nudged elastic band method for finding saddle points and minimum energy paths. J. Chem. Phys. 113, 9901 (2000)

\section{Acknowledgements}

This work is supported by the US Army Research Office MURI grant W911NF-11-10362. N.A. and M.T. acknowledge the Penn State Center for Nanoscale Science support through the 2D Layered Materials seed grant DMR-0820404. X.Z. and B.I.Y acknowledge support by the Robert Welch Foundation (C-1590). We are thankful to R. Lv, L. Pulickal Rajukumar, Z. Lin and C. Zhou for technical assistance and helpful discussions. TEM imaging was performed at NCEM, which is supported by the Office of Science, Office of Basic Energy Sciences of the US Department of Energy under Contract No. DE-AC02-05CH11231. The computations were performed at the Data 
Analysis and Visualization Cyberinfrastructure funded by NSF under Grant OCI0959097. G.S. also acknowledges support from NSF under MRSEC grants DMR-0820404 and DMR-1210588.

\section{Author contributions}

A.A. analysed and processed the data, and performed the geometric phase analysis. X.Z. and Z.Z. carried out the first-principles calculations. P.E. performed the STEM imaging and simulations. A.L.E. and N.P.-L. synthesized the $\mathrm{WS}_{2}$ films and prepared the TEM sample. G.S. did the real space analysis of the strain. All the authors discussed the data presented in the paper.

\section{Additional information}

Supplementary Information accompanies this paper at http://www.nature.com/ naturecommunications

Competing financial interests: The authors declare no competing financial interests.

Reprints and permission information is available online at http://npg.nature.com/ reprintsandpermissions/

How to cite this article: Azizi, A. et al. Dislocation motion and grain boundary migration in two-dimensional tungsten disulphide. Nat. Commun. 5:4867 doi: 10.1038/ ncomms5867 (2014). 